\title{
Noninteger Dimensional Spaces and the Inverse Square Law
}

\author{
Subhash Kak \\ Oklahoma State University, Stillwater
}

\begin{abstract}
Noninteger dimensionality, which shows up in many fields of physics and engineering, is generally viewed from the perspective of fractals and measured by the Hausdorff dimension. Motivated by information theoretic considerations and by extending the principle of complementarity, we propose a new interpretation in which it engenders a potential. The inverse square law may then be seen to originate from the properties associated with the noninteger dimensional space, and it further indicates a relationship between the dynamics and the actual value of the dimensions that may be used for experimental test of the interpretation.
\end{abstract}

\section{Introduction}

The dimension of a mathematical space is the minimum number of coordinates, or bins, needed to specify any point within it. But since surfaces and solids may be scanned into one-dimensional data, one can say that the count of dimensions is ultimately tied to intuition and the very nature of cognitions [1][2]. Space is taken to be three-dimensional in physics for that seems the most obvious thing to do, and the idea of the four dimensions of spacetime is that events are not absolutely defined spatially and temporally, but rather are relative to the motion of the observer. Dimensions also apply to abstract parameter or configuration spaces such as in Lagrangian or Hamiltonian mechanics.

The three-dimensionality of the common intuition in terms of length, breadth, and height is a consequence of our experience, so it is imperative to put it to test. We know that under certain conditions, it becomes necessary to go beyond three to noninteger dimensions, which are used in various branches of science to explain confinement in certain systems [3], the emergence of scale invariant and fractal phenomena such as turbulence [4], human physiology, medicine, and neuroscience [5][6], and engineered networks [7]. The most commonly used measures are the box-counting, information, packing, and the Hausdorff dimensions [8]. These measures are useful indices to describe sets, and it appears reassuring that for ordinary geometric shapes, they equal the familiar Euclidean or topological dimension. In general, these dimensions appear to measure the apparent density of the given set, and in conformity with commonsense a noninteger space is less dense than the one with the immediately higher integer. 
In some theories, noninteger dimensions are introduced through dimensional regularization. Since Gaussian integrals may be written as a $d$-dimensional product of a single dimensional Gaussian integral, one can generalize them to noninteger dimensions. The continuous squeezing of a three-dimensional space into a twodimensional space also generates intermediate scenarios with noninteger dimensions [9]. They are introduced elsewhere to address the origins of self-similar structures [10][11], where generally the Hausdorff dimension is used to fit the experimental data.

In a system with the subject/object divide, the two sides may have descriptions that are formally incompatible with each other. From an epistemological perspective, the complementarity principle provides a way to declare both such descriptions as two sides of the same reality. Since the notion of dimension applies to physical reality at all conceivable scales, one must consider both the objective view in terms of the physical location of things within the space, and the subjective view in terms of the motions of the objects as defined by an underlying dynamic that leads to the locations.

This paper extends recent work showing that optimal representation is e-dimensional and that this idea may apply to physical space itself [12] [13]. We present background material on Hausdorff dimensions of certain recursive sets. Next, we summarize earlier work on e-dimensionality and then, using the principle of complementarity, introduce a new way to interpret noninteger dimensionality and investigate its properties and implications.

Whereas in current understanding, a potential is assumed to exist a priori and then the inverse square law emerges by considering the field across an equipotential sphere of radius $r$ with surface of $4 \pi r^{2}$, we propose that that the origin of the potential lies in the noninteger nature of the dimensions. Specifically, we demonstrate that the new interpretation leads to the dynamics of the inverse square laws, together with behavior that can be put to experimental test.

\section{Background}

The formal use of three-dimensional space in mathematical theory may be traced back to the beginnings of modern physics. Newton's Principia, which formulated the classical picture of reality, describes time, space, place, and motion in two ways: first, in the relative conception of them, and, second, in the absolute, true, mathematical quantities themselves: "Absolute, true, and mathematical time, from its own nature, passes equably without relation to anything external, and thus without reference to any change or way of measuring of time; [and] absolute, true, and mathematical space remains similar and immovable without relation to anything external."[14] Although 
not explicitly defined, absolute time was supposed to flow uniformly and absolute space was taken to be three-dimensional. This was extension of the Aristotelian view of the universe as a container in which the sun, the moon, planets and stars are embedded in perfectly concentric crystal spheres that rotate at fixed rates. The motion of material objects was ultimately conceived with respect to absolute time and space.

Much before Newton, Aristotle took the terrestrial objects to be composed of four elements that rise or fall. The earth, the heaviest element, and water, fall toward the center of the universe; hence the earth and the oceans constitute our planet. At the opposite end, the lightest elements, air and fire, rise up and away from the center. This was the "scientific" reason why the earth was viewed to be at the center of the solar system, and the universe.

In the observer-centric Indian physics tradition, the beginnings of which are traced to Kanāanda, it is asserted that physical laws must be based only on substances, their properties, and their motion [15], but the experience of time and space is a consequence of the relation between the observer and the system being observed. It is an interesting fact that Nicola Tesla [16] knew of this tradition, and through him it may have influenced mainstream physics of early twentieth century. In general, this position is in accord with the notion that abstractions are a creation of the mind, and not independent entities in themselves.

Leibniz proposed a different view of time in which events are more fundamental than time instants. According to him, the primary role is to be ascribed to matter and its properties and it is in relation to these properties that one can speak of time and space. Time is simply succession of instantaneous configurations of matter and not something that flows independent of the bodies in the universe [17]. Mach developed Leibniz's ideas by postulating that only concepts that have an empirical basis should be used in physics, and this eventually led to the theory of relativity and the notion of spacetime. Nevertheless, potentials were assumed to have an a priori existence.

A space consists of objects that are treated as points, and selected relationships between these points. Mathematical spaces are assumed to be dense and metrically unbounded. Given points $x, y, z$ in a space, the distance metric satisfies the axioms $d(x, y)=0$ iff $x=y, d(x, y)=d(y, x)$, and the triangle inequality

$$
d(x, y) \leq d(x, z)+d(z, y)
$$


The distance metric in the Euclidean three-dimensional space is $d(x, y)=$ $\sqrt{\sum\left(x_{i}-y_{i}\right)^{2}}$, where $x_{i}$ and $y_{i}$ are the coordinates of $x$ and $y$ in three orthogonal directions. In the general non-Euclidean case, the distance may be given by the form $d s^{2}=g_{\mu \nu} d x^{\mu} d x^{\nu}$, where sum over repeated indices is implied, and the indices $\mu, v$ run over four spacetime coordinates. The coefficient $g_{\mu \nu}$, which is a function of the spacetime coordinates $x^{\mu}$, represents the metric and it specifies the geometry of the spacetime with respect to the underlying theory.

In general relativity, one assumes some particular distribution of energy and matter in the universe to obtain $T_{\mu \nu}$, the energy-momentum tensor of the matter present. Given a particular $T_{\mu \nu}$, the Einstein equation turns into a system of second-order nonlinear differential equations whose solutions give us the metric $g_{\mu \nu}$. There are also attempts at tacking on extra dimensions to unify forces, but these efforts have not been successful.

In this paper we consider analysis at the more fundamental level of information and structure. Topological characteristics of space must be consistent with the overarching logical framework joining the observer and the system and we must specify measures that are used to characterize the dimensions of a space. Let $S$ be a subset of a metric space $\mathrm{X}$, then its Hausdorff dimension $D(S)$ is the infimum of $d \geq 0$ such that the $d$ dimensional Hausdorff measure of $S$ is 0 (which need not be an integer). It is a measure of the density of the space as we will see in many examples that follow.

Informally, using the box-counting dimension of a set $S$ in a Euclidean space $\mathbf{R}^{n}$, suppose that $N(\varepsilon)$ is the number of boxes of side length $\varepsilon$ required to cover the set represented by the scaling law. Then the box-counting dimension of the scaling law (equal in our examples to Hausdorff dimension of noninteger value) is defined as:

$$
D_{\text {box }}(S)=\lim _{\epsilon \rightarrow \infty} \frac{\log N(\epsilon)}{\log \left(\frac{1}{\varepsilon}\right)}
$$

The examples below are finite, but since the size of the line segments is not specified and it can be as large as one likes, we are, in effect, considering infinite sets.

Example 1. Consider the one-dimensional asymmetric Cantor set. In this, at each stage, the quarter to the left of the midpoint is removed (Figure 1). 


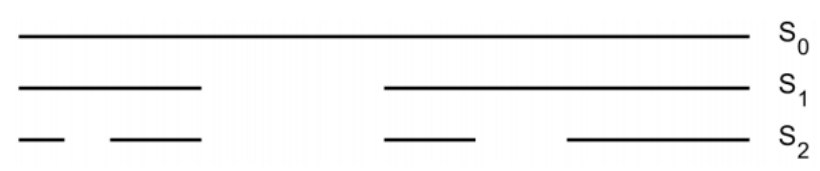

Figure 1. Asymmetric Cantor set

There are two relative length scales of $\lambda_{a}=1 / 4$, and $\lambda_{b}=1 / 2$. Let $N_{a}(\varepsilon)$ and $N_{b}(\varepsilon)$, be the number of boxes of size $\varepsilon$ needed to cover $\lambda_{a}$ and $\lambda_{b}$, respectively.

$$
\begin{aligned}
& N_{b o x}(\varepsilon)=N_{a}(\varepsilon)+N_{b}(\varepsilon) \\
& A \varepsilon^{-D}=A\left(\frac{\varepsilon}{\lambda_{a}}\right)^{-D}+A\left(\frac{\varepsilon}{\lambda_{b}}\right)^{-D} \\
& 1=\lambda_{a}^{D}+\lambda_{b}^{D}
\end{aligned}
$$

Substituting $\lambda_{a}=1 / 4, \lambda_{b}=1 / 2$, and solving the quadratic equation, one obtains:

$$
\left(\frac{1}{4}\right)^{D}+\left(\frac{1}{2}\right)^{D}-1=0 ; \text { or } \frac{1}{2^{D}}=\frac{-1+\sqrt{5}}{2}
$$

Therefore, the Hausdorff dimension, D, of the asymmetric Cantor set is:

$$
D=-\frac{\ln \left(\frac{-1+\sqrt{5}}{2}\right)}{\ln 2} \approx 0.6942
$$

The symmetric Cantor set, in which at every step we leave out the middle-third (Figure 2 ), may be taken as a special case of the above where $\lambda_{a}=\lambda_{b}=1 / 3$. Or $2(1 / 3)^{D}=1$. In other words, $3^{D}=2$, a consequence of the fact that the iteration implies leaving out one part for every three. Or,

$$
D=\frac{\ln 2}{\ln 3} \approx 0.6309
$$

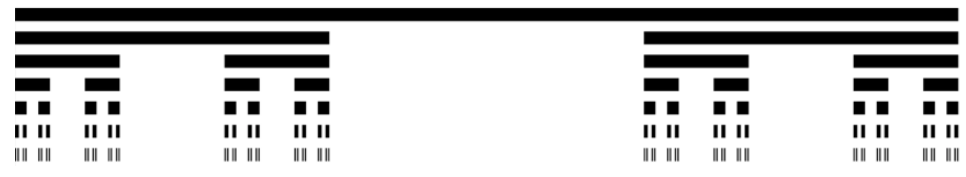

Figure 2. The symmetric Cantor set through several iterations

That the dimension for the symmetric set is less than that for the asymmetric test appears reasonable since one is removing only $25 \%$ of the set in each iteration for the 
asymmetric set as compared to the $33 \%$ for the symmetric one, although the difference in the dimensions is not according to this exact proportion.

Example 2. In the Koch curve, we begin with a straight line, and divide it into three equal segments and replace the middle segment by the two sides of an equilateral triangle of the same length as the segment being removed. This is repeated, taking each of the four resulting segments, dividing them into three equal parts and replacing each of the middle segments by two sides of an equilateral triangle, and so on.

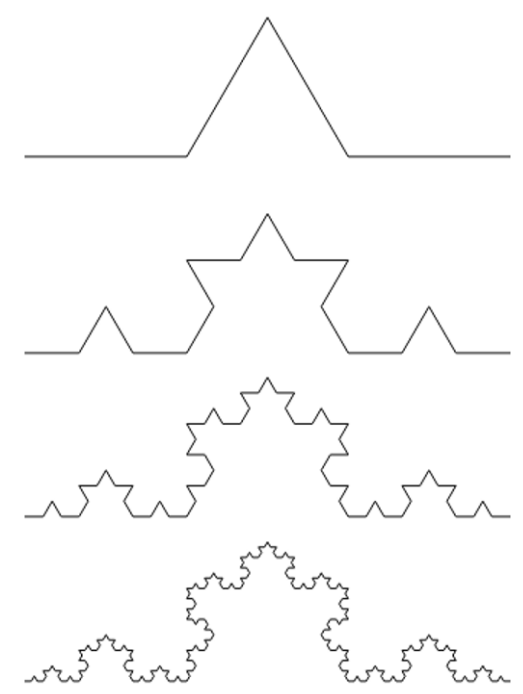

Figure 3. Koch Curve through four iterations

Similar to the computation for the symmetric Cantor set, the dimension of the Koch curve is:

$$
D=\lim _{n \rightarrow \infty} \frac{\ln 4^{n}}{\ln 3^{n}}=\frac{\ln 4}{\ln 3} \approx 1.2619
$$

This is exactly twice that of the symmetric Cantor set.

Example 3. Generalizing the Cantor set to two dimensions, each cell may be transformed according to the rule of the Sierpiński carpet mapping in which we began with a black square of unit length, and at the first iteration we have 9 sub-squares of length $1 / 3$ each, of which the middle one is removed, leaving us with 8 black subsquares and one white sub-square. At the second iteration, this process is applied to each of the 8 black sub-squares as shown in Figure 4 . 

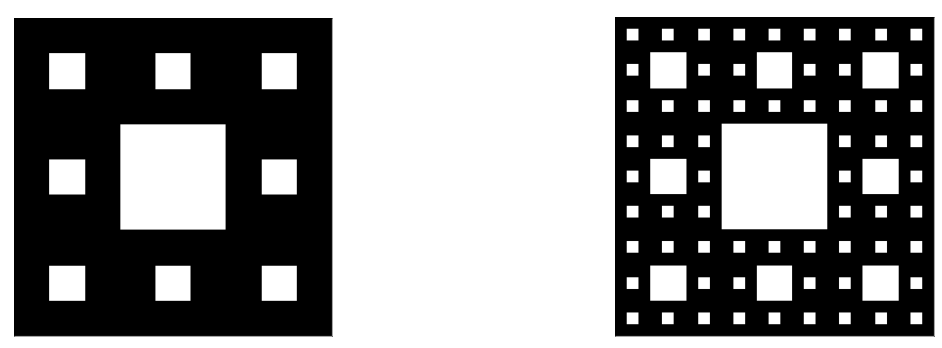

Figure 4. Iterative mapping of the Sierpiński carpet ( $2^{\text {nd }}$ and $3^{\text {rd }}$ iterations)

Since the process replaces 9 sub-squares by 8 with the scaling of $1 / 3$, the Hausdorff dimension of the Sierpiński carpet is $D=\frac{\log 8}{\log 3} \approx 1.8928 \ldots$

Example 4. A further generalization of the Sierpiński carpet leads to the Menger sponge [18]. Since a cube has six sides, this may be done by any mapping where one dark subcube is removed from each of the six sides together with the one at the center.

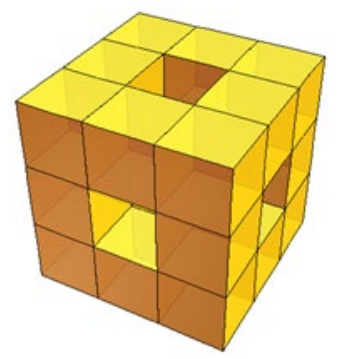

Figure 5. A mapping of 20 e-space sub-cubes into the unit cube

Let $N_{n}$ be the number of dark boxes, $L_{n}$ the length of a side of a light sub-cube, and $V_{n}$ the fractional volume of the dark cubes after the $n$th iteration, then

$$
\begin{aligned}
& N_{n}=20^{n} \\
& L_{n}=\left(\frac{1}{3}\right)^{n}=3^{-n} \\
& V_{n}=\left(\frac{20}{27}\right)^{n}
\end{aligned}
$$

The dimension of such an iterative system is:

$$
\begin{aligned}
& D=\lim _{\varepsilon \rightarrow \infty}\left(-\frac{\log N_{n}}{\log L_{n}}\right) \\
& =\frac{\ln 20}{\ln 3} \approx 2.7268 \ldots
\end{aligned}
$$


which is quite close to $\mathrm{e}=2.71828 \ldots$ We mention this closeness since informationtheoretic considerations [12][13] that are summarized below lead to the conclusion that $e$-dimensional representation is optimal.

Note that for any comparison with natural systems the iteration algorithm must be translated to a physical theory-based evolution equation. Even with such an equation, the fundamental uncertainty in nature will make the evolution random rather than the deterministic one of the above examples. But it must be added that random versions of the algorithms described in the examples above provide more natural-like distributions.

\section{Information theoretic view of dimensionality}

Consider the logical foundations of the conception of a $d$-dimensional space. On grounds of maximization of information, one must assume that each of the dimensions has the same probability or, in other words, the space is uniform. The probability of each of the dimensions is $1 / d$, and the information associated with each is $\ln d$, so that information efficiency per dimension is $E(d)=\frac{\ln d}{d}$ (Figure 6 shows the efficiency for $d$ between 2 and 4). Its maximum value is obtained by taking the derivative of $E(d)$ and equating that to zero, which yields $d_{\text {optimum }}=e=2.71828$... The value of dimensional efficiency beyond 4 falls rapidly.

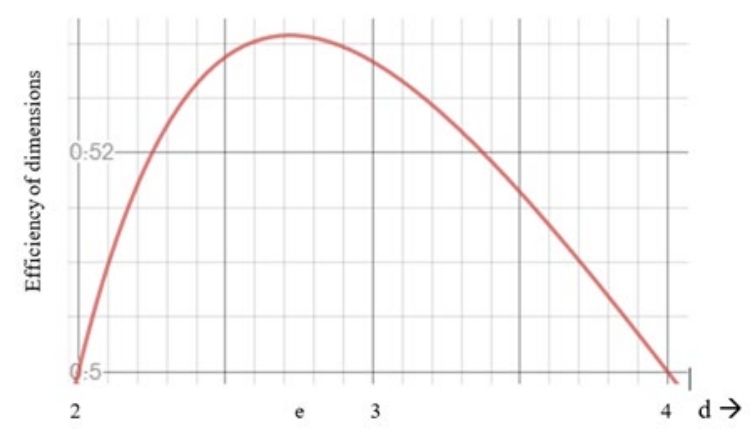

Figure 6. Efficiency of dimensions for $d$ between 2 and 4, with peak at $e$

Nature is optimal and it does all with the utmost economy and this optimality is one way to discover physical laws. Since the most efficient representation of information is to base-e, we may conclude that space is e-dimensional, or that Nature does it work using base-e arithmetic. Also, dimensions are additive, and so, on grounds of equivalence, we must accept that physical space is uniform in all directions, and the fraction $\frac{e}{3}$ will apply in any random direction. 
The distance metric in a noninteger space may be seen through the prism of the dynamics associated with such a space. Further elaboration is required to determine the relationship of the geodesic lines between points in the presence of matter, and also of behavior with the value of the noninteger dimension changing as the space is progressively squeezed.

\section{Complementarity}

The complementarity principle holds that objects have pairs of complementary properties that cannot be observed or measured simultaneously. As a principle, it provides a philosophical basis for why in certain situations a photon is a particle whereas in other situations it is a wave. The idea is that reality is not one or the other but manifests its properties based on how we interact with it. In general, the complementarity is across conjugate variables that constitute transform pairs.

The complementarity that we consider here is between two views: (i) the noninteger space sits within the container of the ceiling integer space (e.g. e-dimensional space sits within the 3-dimensional space), and (ii) noninteger space is characterized by a continual shrinking of the metrical relationships between objects.

Complementarity becomes relevant when we consider large scale structure of the universe. The set consisting of the locations of stars and galaxies may be viewed from the perspective of the distribution of the locations (and this is not sufficient since different histories may intersect) and how they have arrived at their locations. As an application of this idea the discrepancy [19] between the early and late universe estimates of the Hubble constant may be explained away if one recognizes that the early universe model provides the true e-dimensional estimate whereas the late universe model imposes a 3-dimensional gloss on the measurement [20].

\section{Dynamics}

Consider the example of a noninteger space with dimension $d$. We need to compare it with its ceiling function $\lceil d\rceil$, and see what it means along the one-dimensional infinite line in any direction. The noninteger dimension can either mean that there are gaps in the space (as illustrated by the earlier examples) or it may mean that relative measures on the line tend to shrink in proportion to the value of dimensionality, and the tendency to do so is in the ratio $\frac{(\lceil d\rceil-d)}{\lceil d\rceil}$. Thus an $e$-dimensional space will tend to shrink by $\frac{(3-e)}{3} \approx$ 0.94 in each direction. We don't argue for one over the other in the two complementary views, and the interpretation that is appropriate will depend on the context. 
The tendency for space to contract constitutes a potential, making points associated with events that the observer witnesses to tend to come closer to each other. This potential leads to dynamics that emerge thus from the very nature of space. This potential is not assumed to have an a priori existence, independent of dimensions, and therefore it represents a view different from the current understanding. Note, the potential for integer dimensional spaces is zero.

Let's explore the situation further. Consider two objects with measures $m_{A}$ and $m_{B}$ with a separation of $r$.

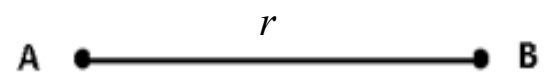

Figure 7. Two objects separated by $r$ experiencing a potential

Since $d$ characterizes the space, the potential between two objects with unit measures and separation of $r$ will tend to shrink this separation into $\frac{(\lceil d\rceil-d) r}{\lceil d\rceil}$, or the potential is proportional to $\frac{(\lceil d\rceil-d) r}{\lceil d\rceil}$. The potential must also be inversely related to the separation so that objects that are further apart have less influence on each other than those that are near. Since the sphere representing the equipotential surfaces at a distance of $r$ has surface equal to $4 \pi r^{2}$, the potential will be proportional to $\frac{(\lceil d\rceil-d) r}{\lceil d\rceil} \times \frac{1}{4 \pi r^{2}}=\frac{(\lceil d\rceil-d)}{4 \pi\lceil d\rceil r}$. The potential is proportional to the difference from the ceiling integer value, and the potential for $d=2.9$ is one-fifth of that for $d=2.5$.

The measures $m_{A}$ and $m_{B}$ of objects $\mathrm{A}$ and $\mathrm{B}$ represent the attributes of the objects and, assuming linearity, which is reasonable, each component of $A$ sets up a potential with respect to each component of $B$, and vice versa. Summing up all these components implies a product of the two measures. For the general case of a $d$ dimensional infinite space, the potential $U_{A B}$ is:

$$
U_{A B}=\frac{-k(d) m_{A} m_{B}}{r}
$$

where $k$ is the constant of proportionality that depends on the properties of the objects $\mathrm{A}$ and $\mathrm{B}$ :

$$
k(d) \propto \frac{(\lceil d\rceil-d)}{4 \pi\lceil d\rceil}
$$


Purely for a figurative comparison with a special Cantor set, we visualize two objects $A$ and $B$ anchored at a separation of $r$ units, each of which is an accumulation of many smaller objects half of which are free to move. The potential working on each of them is shown by the straight line joining them. The force between the two objects leads to a portion of $A$ reaching $D$, and likewise a portion of $B$ reaching $E$. If now $D$ and $E$ are also anchored with half free, then in the second iteration, leaving out the possible interaction between $D$ and $E$ because they are much further apart, we get the situation of Figure 8.

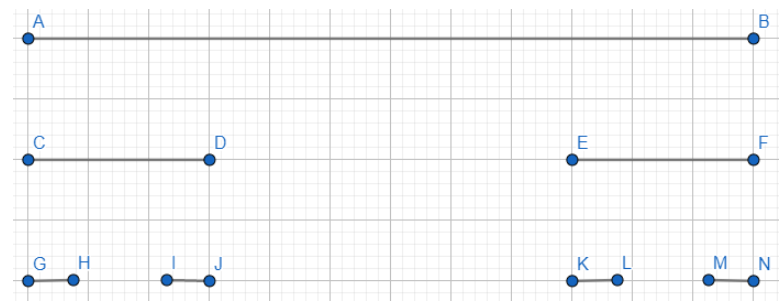

$1^{\text {st }}$ iteration

$2^{\text {nd }}$ iteration

Figure 8. Dynamics associated with the potential

This provides a simple cartoon-like pictorial description of the dynamic underpinning to the Cantor set generation process.

\section{Inverse square laws}

Inverse-square laws (such as Newton's gravitation law or Coulomb's electrostatic law) are experimental laws that have been subject to much testing to see if the formulas are exact. The gravitation law can be derived within General Relativity in the nonrelativistic limit in a weak gravitational field. Its validity has been verified experimentally to $95 \%$ confidence level down to distances of less than $1 \mathrm{~mm} \mathrm{[21]} \mathrm{and} \mathrm{deviations} \mathrm{examined} \mathrm{at}$ the micrometer and $\mathrm{nm}$ range [22] [23].

As an amusing sidelight to the history of the Coulomb's Law [24], John Robison announced in 1769 that, according to his measurements, the force of repulsion between two spheres with charges of the same sign varied according to the exponent -2.06. The logic of the inverse square law is based on the fact that the surface of a sphere varies with the square of the radius $r$, and so the exponent should be exactly 2 ; it is to confirm this logic that various modern studies have been done to test the law [25].

Considering the potential of equation (12), the force, F, associated with the potential will be given by

$$
F_{A B}=\frac{d U}{d r}=\frac{k(d) m_{A} m_{B}}{r^{2}}
$$


The actual dependence of $k(d)$ on $d$ may reveal behaviors that are of potential use in engineered systems. Another important problem to investigate is the implication for angular motion. The dynamics in a finite space may be different because of boundary conditions.

It is possible that whereas overall dimensionality of space is $e$ across the universe, it is distributed in a random fashion across different regions. If that were the case, the constants of the inverse laws will vary across regions, with implications for the largescale structure of the universe.

\section{Conclusions}

Motivated by the mathematical result that $e$-dimensionality is optimal, the paper explored some questions associated with dynamics for the broader class of noninteger dimensional spaces found in natural and engineered systems. A novel interpretation of noninteger dimensionality was used to derive the inverse square law. Whereas in the standard understanding, a potential is assumed to exist a priori and then the inverse square law emerges by considering the field across an equipotential sphere of radius $r$, we proposed that that the origin of the potential lies in the noninteger nature of the dimensions.

Specifically, we demonstrated that the new interpretation leads to behavior that can be put to experimental test. If the dimensionality dependence of the constant $k$ associated with the law is confirmed by experiment, it will change current understanding of noninteger spaces and the nature of the inverse square law.

\section{REFERENCES}

1. Gautam, A. and Kak, S. Symbols, meaning, and origins of mind. Biosemiotics 6, 3, 301309 (2013)

2. Kak, S. Communication languages and agents in biological systems. In Biocommunication: Sign-Mediated Interactions between Cells and Organisms, R. Gordon and J. Seckbach (editors). World Scientific Publishing, London, 203-226 (2016)

3. Garrido, E., Jensen, A.S., Confinement of two-body systems and calculations in d dimensions Phys. Rev. Research 1, 023009 (2019)

4. Sreenivasan, K.R. Fractals and multifractals in fluid turbulence. Annual Rev. Fluid Mech. 23, 539-600 (1991)

5. Losa, G.A., Nonnenmacher, T. F., eds. Fractals in biology and medicine. Springer (2005) 
6. Kak, A., Gautam, A., Kak, S. A three-layered model for consciousness states. NeuroQuantology 14, 166-174 (2016)

7. Kak, S. Power series models of self-similarity in social networks. Information Sciences 376, 31-38 (2017)

8. Mandelbrot, B. B. The Fractal Geometry of Nature. W. H. Freeman (1983)

9. Stillinger, F.H. Axiomatic basis for spaces with noninteger dimensions. Journal of Mathematical Physics 18, 1224- 1234 (1977).

10. Falconer, K.J. Fractal Geometry: Mathematical Foundations and Applications. Wiley (2003)

11. Bunde, A., Havlin, S. Fractals in Science. Springer Science \& Business Media (2013)

12. Kak, S. The base-e representation of numbers and the power law. Circuits Syst. Signal Process. (2020) https://doi.org/10.1007/s00034-020-01480-0

13. Kak, S. Information, representation, and structure. International Conference on Recent Trends in Mathematics and Its Applications to Graphs, Networks and Petri Nets, New Delhi, India (2020) https://www.techrxiv.org/articles/preprint/Information_Representation_and_Structu re/12722549

14. Newton, I. Philosophiae Naturalis Principia Mathematica, Bk. 1, 1689; Florian Cajori (ed.) University of California Press (1934)

15. Kak, S. Matter and Mind. Mount Meru Publishing (2016)

16. Kak, S. Tesla, wireless energy transmission and Vivekananda. Current Science 113, 2207-2209 (2017)

17. Arthur, R. Space and Relativity in Newton and Leibniz. British Journal for the Philosophy of Science, 45, 219-240 (1994)

18. Menger, K. Reminiscences of the Vienna Circle and the Mathematical Colloquium. Springer Science \& Business Media (2013)

19. Panek, R. A cosmic crisis. Scientific American 322, 3, 30-37 (2020)

20. Kak, S. Geometry of numbers and dimensionality. (2020) https://www.techrxiv.org/articles/preprint/Geometry_of_numbers_and_dimensionali ty/12786356

21. Adelberger, E.G., Gundlach, J.H., Heckel, B.R. et al. Torsion balance experiments: A low-energy frontier of particle physics. Progr. Particle Nuclear Phys. 62, 102 (2009)

22. Haddock, C.C., Oi, N., Hirota, K. et al. Search for deviations from the inverse square law of gravity at nm range using a pulsed neutron beam. Physical Review D 97, 6, id.062002 (2018)

23. Tan, w.-H., Du, A.-B., Dong, W.-C. et al. Improvement for testing the gravitational inverse-square law at the submillimeter range. Phys. Rev. Lett. 124, 051301 (2020)

24. Robison, J. A System of Mechanical Philosophy. London (1822)

25. Tu, L.-C. and Luo, J. Experimental tests of Coulomb's Law and the photon rest mass. Metrologia 41, 5 (2004) 